Publ. RIMS, Kyoto Univ.

12 Suppl. (1977), 267-300.

\title{
Introduction to Microlocal Analysis
}

\author{
by
}

Tetsuji MiwA*, Toshio OshImA** and Michio JIMBO*

\section{$\S 1$. Hyperfunctions, Microfunctions and the Exact Sequence of Sato}

The most important example of a hyperfunction is the $\delta$-function of Dirac. It can be characterized up to constant multiple by the differential equation

$$
\left(x D_{x}+1\right) f(x)=0,
$$

and the support property

$$
f(x)=0 \quad \text { if } x>0 \text { or } x<0 .
$$

Even if we omit the condition (1.2), we cannot find any real analytic function other than zero which satisfies $(1 \cdot 1)$ in a neighborhood of zero. Therefore it is necessary to generalize the concept of functions.

If we consider $(1 \cdot 1)$ in the complex plane, its solution is

$$
F(z)=\text { const. } \frac{1}{z}
$$

It is holomorphic in the upper half plane and also in the lower half plane. If we can attach some meaning to the phrase "the boundary value of $F(z)$ from upper (lower) half plane", we can find non trivial solutions of $(1 \cdot 1)$. These are hyperfunctions

$$
\frac{1}{x+i 0}
$$

and

$$
\frac{1}{x-i 0}
$$

Received October 12, 1976.

* Research Institute for Mathematical Sciences, Kyoto University, Kyoto 606, Japan. ** Department of Mathematics, Faculty of Science, The University of Tokyo, Japan. 
These two solutions form a basis of the solution space of $(1 \cdot 1)$. Now we can find a solution which satisfies (1.2) taking the difference of $(1 \cdot 4)$ and (1.5). Adjusting the constant, we have the well-known formula

$$
\delta(x)=-\frac{1}{2 \pi i}\left(\frac{1}{x+i 0}-\frac{1}{x-i 0}\right)
$$

Thus $\delta(x)$ can be expressed as a sum of two boundary values.

Definition 1.7. A hyperfunction is a sum of real boundary values of holomorphic functions.

To give rigorous and mathematical meaning to the above definition we use cohomology theory, the theory of residue class. (It is only a sky-scraper made of the same material which we use to construct rational numbers from integers.) In the case of one independent variable, the space of hyperfunctions in an open set $U$ in $\boldsymbol{R}$ is defined by

$$
\mathscr{B}(U)=\left(\mathcal{O}\left(V_{+}\right) \oplus \mathcal{O}\left(V_{-}\right)\right) / \mathcal{O}(V) .
$$

Here $V$ is a complex neighborhood of $U$ which contains $U$ as a closed subset, and $V_{1}\left(V_{2}\right)$ is the intersection of $V$ and the upper (the lower) half plane. (Fig. 1.9)

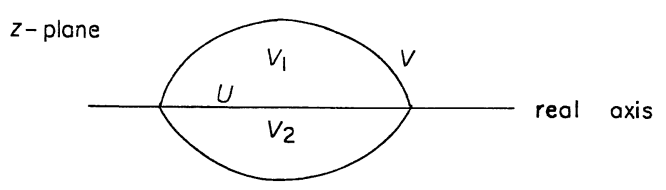

Fig. 1.9

If $F_{1}(z)$ is holomorphic in $V_{1}$ we write the hyperfunction defined as the boundary value of $F_{1}(z)$ like

$$
F_{1}(x+i 0) \text {. }
$$

Similarly we define a hyperfunction

$$
F_{2}(x-i 0)
$$

A hyperfunction $u(x)$ has an expression

$$
f(x)=F_{1}(x+i 0)+F_{2}(x-i 0) .
$$


If $\tilde{f}(x)=\widetilde{F}_{1}(x+i 0)+\widetilde{F}_{2}(x-i 0)$ is another hyperfunction, $f(x)=\tilde{f}(x)$ if and only if

$$
\begin{aligned}
& F_{1}(x+i 0)-\widetilde{F}_{1}(x+i 0) \in \mathcal{A}, \\
& F_{2}(x-i 0)-\widetilde{F}_{2}(x-i 0) \in \mathcal{A},
\end{aligned}
$$

and

$$
\left(F_{1}(x+i 0)-\widetilde{F}_{1}(x+i 0)\right)+\left(F_{2}(x-i 0)-\widetilde{F}_{2}(x-i 0)\right)=0 .
$$

We can summarize $(1 \cdot 12) \sim(1 \cdot 15)$ in the following exact sequence - the exact sequence of Sato.

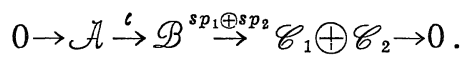

In $(1 \cdot 16) \mathscr{C}_{1}\left(\mathscr{C}_{2}\right)$ is the space of the element $(1 \cdot 10)((1 \cdot 11))$ where two elements like $(1 \cdot 13)((1 \cdot 14))$ are identified.

Exercise. 1.17 Examine that differential operators operate on $\mathscr{B}$, $\mathscr{C}_{1}$ and $\mathscr{C}_{2}$ as local operators.

We have characterized $\delta(x)$ by $(1 \cdot 1)$ and $(1 \cdot 2)$. To characterize $(1 \cdot 4)$ and $(1 \cdot 5)$ we can use $(1 \cdot 16)$. (1.4) $((1 \cdot 5))$ is an element in $\mathscr{B}$ whose image under $s p_{2}\left(s p_{1}\right)$ vanishes. We can illustrate these three cases of "support property" by the following diagram. (Fig. 1.18).

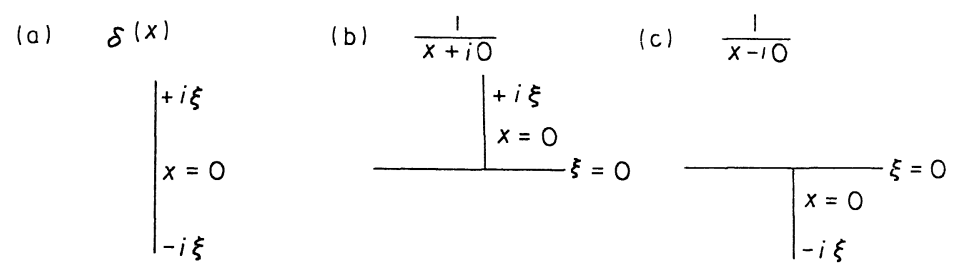

Fig. 1.18 The notation $\pm i \xi$ will be understood when we explain the case of several independent variables.

Remark. 1.19 The diagram for a general hyperfunction is not so simple. Nevertheless it is important to note that a kind of hyperfunction as $F(x+i 0)(F(x-i 0))$ has no support below (above) the axis $\{\xi=0\}$.

Before proceeding to the case of several independent variables, we give several examples of hyperfunctions with one independent variable. We consider a differential equation with one complex parameter $\lambda$. 


$$
\left(x D_{x}-\lambda\right) u=0 \text {. }
$$

Two independent solutions of $(1 \cdot 20)$ are

$$
(x+i 0)^{\lambda}
$$

and

$$
(x-i 0)^{2}
$$

for $\lambda \neq 0,1,2, \cdots$. Here we choose the branch such that $z^{\lambda}=1$ for $z=1$. The diagrams for $(x+i 0)^{2}$ and $(x-i 0)^{2}$ are (b) and (c) of (1.18). For $\lambda=-1,-2, \cdots$, we can construct a solution of $(1 \cdot 20)$ whose diagram is like (a) of $(1 \cdot 18)$. The solutions are the $(-\lambda+1)$-th derivative of $\delta(x)$

$$
\delta^{(-\lambda+1)}(x), \quad \lambda=-1,-2, \cdots .
$$

In contrast, for $\lambda \notin \boldsymbol{Z}$ we cannot construct such solutions. Instead we can construct two more solutions with characteristic support property. (Fig. 1.24).
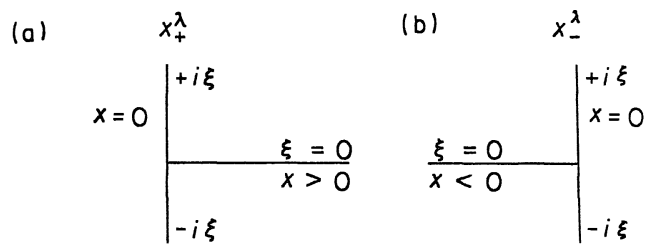

Fig. 1.24

Exercise. 1.25 Express $x_{+}^{\lambda}$ and $x_{-}^{\lambda}$ as linear combinations of $(1 \cdot 21)$ and $(1 \cdot 22)$. Show that they are meromorphic with respect to $\lambda$ and have simple poles at $\lambda=-1,-2, \cdots$. (Hint. Compare the values at $x= \pm 1$, and calculate their Laurent expansions at $\lambda \in \mathbb{Z}$.)

$$
\begin{aligned}
& x_{+}^{\lambda}=\frac{1}{2 i \sin \pi \lambda}\left\{(-x+i 0)^{\lambda}-(-x-i 0)^{\lambda}\right\}, \\
& x_{-}^{\lambda}=\frac{1}{2 i \sin \pi \lambda}\left\{(x+i 0)^{\lambda}-(x-i 0)^{\lambda}\right\} .
\end{aligned}
$$

For $\lambda=0,1,2, \cdots,(1 \cdot 21)$ and $(1 \cdot 22)$ are the same and there is one more independent solution of $(1 \cdot 20)$. Thus we have the following three 
solutions with characteristic support property any two of which form a basis of the solution space of $(1 \cdot 20)$. (Fig. 1.27)

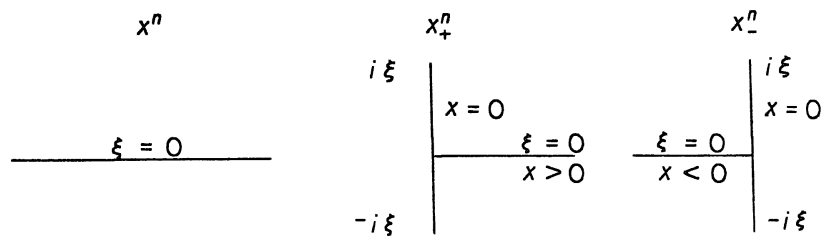

Fig. 1.27

$$
\begin{aligned}
& x_{+}^{n}=-\frac{x^{n}}{2 \pi i}\{\log (-x+i 0)-\log (-x-i 0)\}, \\
& x_{-}^{n}=\frac{x^{n}}{2 \pi i}\{\log (x+i 0)-\log (x-i 0)\} .
\end{aligned}
$$

Summary. 1.30 The dimension of the solution space of $(1 \cdot 20)$ is 2. The structure of support of solutions are classified into three classes.

\section{I. $\lambda \notin \mathbb{Z}$}

II. $\lambda=0,1,2, \cdots$

III. $\lambda=-1,-2, \cdots$.

Later we explain that this difference is due to the difference of algebraic structure of the equations.

Exercise. 1.31 Show that

$$
|x|^{\lambda}=x_{+}^{\lambda}+x_{-}^{\lambda}
$$

is a meromorphic function of $\lambda$ with simple poles at $\lambda=-1,-3, \cdots$. Calculate its Laurent expansion.

To illustrate how the structure of solution space become simple, we state some theorems about more general ordinary differential equations. (The proof is easy, try yourself.)

Theorem 1.33. $P(x, D)=\sum_{\alpha=0}^{m} a_{\alpha}(x) D^{\alpha}$ is an ordinary differential operator defined in a neighborhood $U$ of the origin, or $d_{0} a_{m}$ denotes the 
degree of zero of $a_{m}(x)$ at $x=0$.

(a) $\quad \mathcal{B}(U)^{P} \rightarrow \mathscr{B}(U) \rightarrow 0$ is exact.

(b) $\operatorname{dim} \mathscr{B}(U)^{P}=m+\operatorname{ord}_{0} a_{m}$

where $\mathscr{B}(U)^{P}$ represents the space of hyperfunction solutions in $U$ of the equation $P(x, D) u=0$.

Now we go back to the formula $(1 \cdot 6)$. It is easy to see that the well-known formula

$$
\int f(x) \delta(x-y) d x=f(y)
$$

is equivalent to the Cauchy's integral formula (Fig. 1.35)

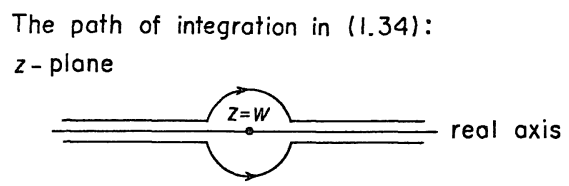

The path of integration in (1.36):

$z$-plane

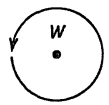

Fig. 1. 35

$$
\frac{1}{2 \pi i} \oint \frac{F(z)}{z-w} d z=F(w) \text {. }
$$

(1.36) tells us every function imaginable can be expressed as a hyperfunction, that is, as a sum of boundary values. In fact, take any "function of one real variable" $f(x)$. We assume its support is contained in the interval $I=[-1,1]$. Then we can define a holomorphic function outside $I$ by

$$
F(z)=\frac{1}{2 \pi i} \int \frac{f(x)}{x-z} d x
$$

Then we have

$$
f(x)=F(x+i 0)-F(x-i 0) \text {. }
$$

Exercise. 1.39 Show that for any real analytic function $g(x)$ 


$$
\int f(x) g(x) d x=\int\{F(x+i 0)-F(x-i 0)\} g(x) d x
$$

This argument can be easily extended to the case of several independent variables using the formula

$$
F\left(w_{1}, \cdots, w_{n}\right)=\frac{1}{(2 \pi i)^{n}} \oint \cdots \oint \frac{F\left(z_{1}, \cdots, z_{n}\right)}{\left(z_{1}-w_{1}\right) \cdots\left(z_{n}-w\right)} d z_{1} \cdots d z_{n} .
$$

Exercise. 1.41 Find the holomorphic region of the function defined by

$$
F\left(z_{1}, \cdots, z_{n}\right)=\frac{1}{(2 \pi i)^{n}} \int \cdots \int \frac{f\left(x_{1}, \cdots, x_{n}\right)}{\left(x_{1}-z_{1}\right) \cdots\left(x_{n}-z_{n}\right)} d x_{1} \cdots d x_{n}
$$

where $f\left(x_{1}, \cdots, x_{n}\right)$ is a function with bounded support.

Thus any hyperfunction can be expressed as a sum of $2^{n}$ boundary values of a holomorphic function which is holomorphic in $2^{n}$ quadrants

$$
V_{\varepsilon_{1} \cdots \varepsilon_{n}}=\left\{\left(z_{1}, \cdots, z_{n}\right) \in C^{n} \mid\left(\operatorname{Im} z_{i}\right) \varepsilon_{i}>0\right\} \quad \text { for } i=1, \cdots, n
$$

where $\varepsilon_{i}= \pm 1$. (Fig. 1.44)

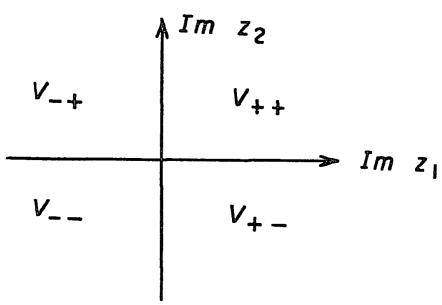

Fig. 1. 44

More generally we can define the boundary value from a tube domain.

Let $U$ be an open set in $\mathbb{R}^{n}$ and $V$ be a complex neighborhood of $U$ in $\boldsymbol{C}^{n}=\boldsymbol{R}^{n}+\sqrt{-1} \boldsymbol{R}^{n}$ which contains $U$ as a closed subset. $V$ is real $2 n$ dimensional and $U$ is embedded in $V$ as a submanifold of real codimensions $n$. Let us consider $n$-dimensional cone $\Gamma$. $\Gamma$ is an open convex subset of $\boldsymbol{R}^{n}$ which is closed under the multiplication by positive real numbers. Then $V \cap(U+\sqrt{-1} \Gamma)$ is a union of $n$-dimensional cones attached to real points in $U$ and is called a tube domain. When we consider 
this tube domain only near $x$ we also denote it by $x+i 0 \Gamma$. We shall use the notation $z=\left(z_{1}, \cdots, z_{n}\right)$. Let $F(z)$ be a holomorphic function in $V \cap(U+\sqrt{-1} \Gamma)$, then we denote by

$$
F(x+i 0 \Gamma)
$$

the boundary value to $U$ of $F(z)$. Here the symbol 0 in front of $\Gamma$ means a residue class of tangent vectors of $X$ at $x$ modulo tangent vectors of the real manifold $U$.

Now we can define a hyperfunction as a sum of boundary values.

$$
f(x)=\sum_{j} F_{j}\left(x+i 0 \Gamma_{j}\right) .
$$

To complete the definition we must define when such expression represents zero. It requires the cohomology theory and we omit it.

Before we proceed on, let us explain some geometrical concepts.

Let $M$ be an $n$ dimensional real analytic manifold and $X$ be its complexification. You may imagine $M=\boldsymbol{R}^{n}$ and $X=\boldsymbol{R}^{n}+\sqrt{-1} \boldsymbol{R}^{n}$. There is an exact sequence which defines a normal vector at $x \in M$.

$$
0 \leftarrow\left(T_{M} X\right)_{x} \leftarrow(T X)_{x} \leftarrow(T M)_{x} \leftarrow 0 .
$$

The dual exact sequence

$$
0 \rightarrow\left(T_{M}^{*} X\right)_{x} \rightarrow\left(T^{*} X\right)_{x} \rightarrow\left(T^{*} M\right)_{x} \rightarrow 0
$$

defines a conormal vector - a cotangent vector of $X$ at $x$ which is zero at tangent vectors of $M$ at $x$. There is a canonical isomorphism

$$
\sqrt{-1} T^{*} M \cong T_{M} * X
$$

$x \in M$ is identified with a zero vector in $\sqrt{-1}\left(T^{*} M\right)_{x} *$ ) Then we define

$$
\sqrt{-1} S^{*} M \cong S_{M}^{*} X
$$

as $\left(\sqrt{-1} T^{*} M-M\right) / \boldsymbol{R}_{+}$and $\left(T_{M}{ }^{*} X-M\right) / \boldsymbol{R}_{+}$(residue class modulo real positive multiple). We denote an element of (1.49) and (1.50) by ( $x$, $i \xi)$ and $(x, i \infty \xi)$. There is a natural projection $\sqrt{-1} T^{*} M \rightarrow M$ and $\sqrt{-1} S^{*} M \rightarrow M$. We denote them by $\pi$. $(x, i \infty \xi)$ represents an infinitesimal half space of $X$ attached to $M$ at $x$. (Fig. 1.51) 


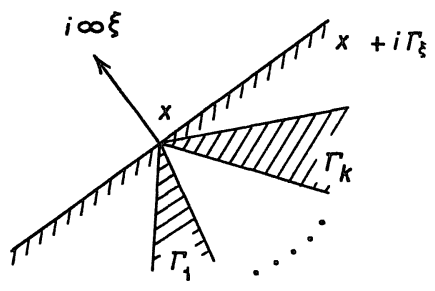

Fig. 1.51

We denote this half space by $x+i \Gamma_{\xi}$.

$$
x+i \Gamma_{\xi}=\left\{x^{\prime}+i 0 y\left|x^{\prime} \approx x,\langle i 0 y, i \infty \xi\rangle=-\langle y, \xi\rangle\right\rangle 0\right\} .
$$

Definition (1.53) (Microanalyticity). A hyperfunction is said to be microanalytic at $(x, i \infty \xi)$ if it can be expressed as a sum of boundary values of holomorphic functions which are holomorphic in tube domains contained in $x+i \Gamma_{\xi}$. (Fig. 1.54)

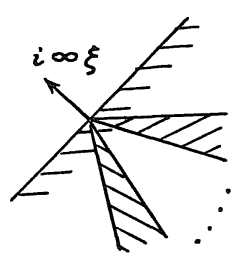

Fig. 1.54

Definition $1.55\left(\mathscr{C}_{(\boldsymbol{x}, i \infty \xi)}\right)$. A residue class of hyperfunctions at $x$ modulo those which are microanalytic at $(x, i \infty \xi)$ defines a microfunction at $(x, i \infty \xi)$.

We collect $\mathscr{C}_{(x, i \infty \xi)}$ to define the sheaf $\mathscr{C}$ of microfunctions. This is a sheaf on $\sqrt{-1} S^{*} M$.

\section{Theorem 1.56. (The exact sequence of Sato)}

$$
0 \rightarrow \mathcal{A} \stackrel{\iota}{\rightarrow} \mathscr{B} \stackrel{s p}{\rightarrow} \pi_{*} \mathscr{C} \rightarrow 0 \text {. }
$$

Here $\pi_{*} \mathscr{C}$ is a sheaf on $M$ whose stalk at $x$ is the set of microfunctions defined on $\pi^{-1}(x)$.

For a hyperfunction $f(x)$, support of $\operatorname{sp} f(x)$ is called the singular

\footnotetext{
* In this sense $M$ is called the zero section of $\sqrt{-1} T^{*} M$.
} 
spectrum of $f(x)$. We denote it by $S . S . f(x) .{ }^{*}$ It is a closed subset in $\sqrt{-1} S^{*} M$ where $f(x)$ is not microanalytic.

Exercise. 1.57 Show that

S.S.F $(x+i 0 \Gamma) \subset\{(x, i \infty \xi) \mid\langle i 0 \Gamma, i \infty \xi\rangle \leqq 0\}$.

Example $1.58 \quad$ S.S. $\delta\left(x_{1}\right) \delta\left(x_{2}\right)$
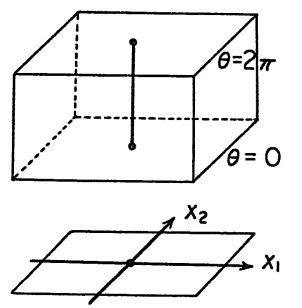

$\sqrt{-1} S^{*} M \supset S^{1}=\pi^{-1}(x)$
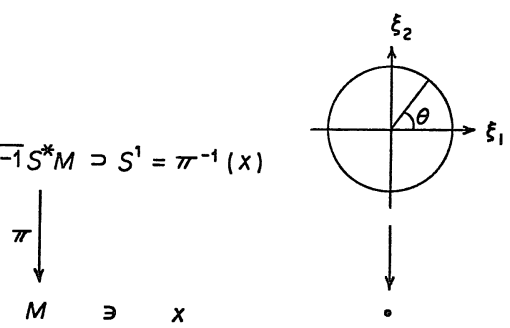

Example 1.59 S.S. $\left(x_{1} \cos \alpha+x_{2} \sin \alpha+i 0\right)^{\lambda}$
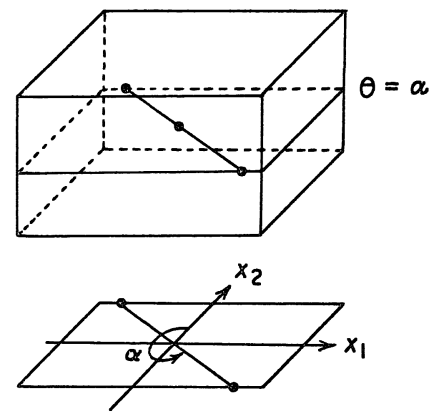

Example 1.60 S.S. $\left\{\left(x_{1} \cos \alpha+x_{2} \sin \alpha\right)+i\left(-x_{1} \sin \alpha+x_{2} \cos \alpha\right)^{2}+i 0\right\}^{\lambda}$
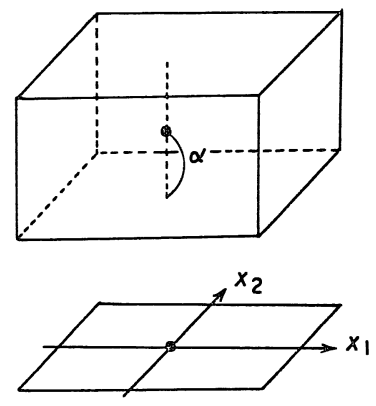

* We also denote by S.S. $f(x)$ the support of a microfunction $f(x)$. A hyperfunction $f(x)$ is real analytic if and only if S.S. $f(x)=\phi$. 
Exercise. 1.61 Show that $\left\{\left(x_{1} \cos \alpha+x_{2} \sin \alpha\right)+i\left(-x_{1} \sin \alpha+\right.\right.$ $\left.\left.x_{2} \cos \alpha\right)^{2}-i 0\right\}^{\lambda}$ cannot be defined.

Example 1.62 S.S. $\left(x_{2}{ }^{2}-x_{1}{ }^{2}-m^{2}+i 0\right)^{\lambda}\left(x_{2}>0\right)$
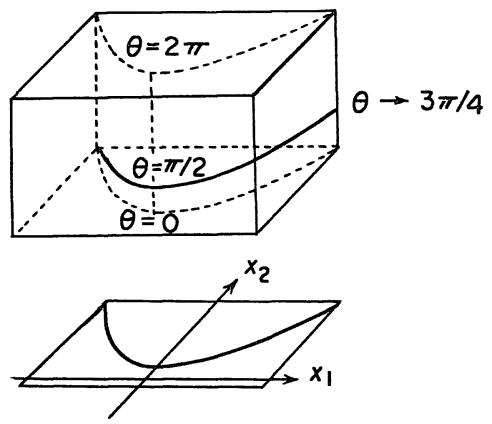

Example 1.63 S.S. $\frac{1}{x_{1}+i 0} \frac{1}{x_{2}+i 0}$
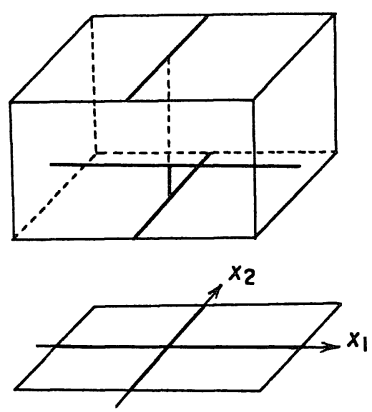

Exercise 1.64 Compare S.S. $\left(\frac{1}{x_{1}+i 0}+\frac{1}{x_{2}+i 0}\right)$ with (1.63).

In examples $(1.59) \sim(1.62)$ each hyperfunction can be expressed as a boundary value from a half space. Therefore its singular spectrum consists of at most one point in $\pi^{-1}(x)$. In contrast, singular spectrum of $\delta\left(x_{1}\right)$. $\delta\left(x_{2}\right)$ spreads all over $\pi^{-1}(0)$. This is concordant with the well-known plane wave expansion of $\delta(x)$.

$$
\delta(x)=\frac{(n-1) !}{(-2 \pi i)^{n}} \int_{S^{n-1}} \frac{\omega_{n-1}(\xi)}{(\langle x, \xi\rangle+i 0)^{n}}
$$

where $\langle x, \xi\rangle=x_{1} \xi_{1}+\cdots+x_{n} \xi_{n}$ and $\omega_{n-1}(\xi)$ is the surface element on $S^{n-1}$. Let $\Gamma_{1}$ and $\Gamma_{2}$ be cones such that $\Gamma_{1} \cup \Gamma_{2}$ is convex. Then Cousin's 
lemma in the theory of functions with several complex variables asserts that there is a decomposition

$$
F\left(x+i 0\left(\Gamma_{1} \cap \Gamma_{2}\right)\right)=F_{1}\left(x+i 0 \Gamma_{1}\right)+F_{2}\left(x+i 0 \Gamma_{2}\right) .
$$

We can proceed with this decomposition until $\Gamma$ reaches at a half space. (Fig. 1.67). From this point of view Exercise (1.57) and Definition $(1 \cdot 53)$ may be well understood.
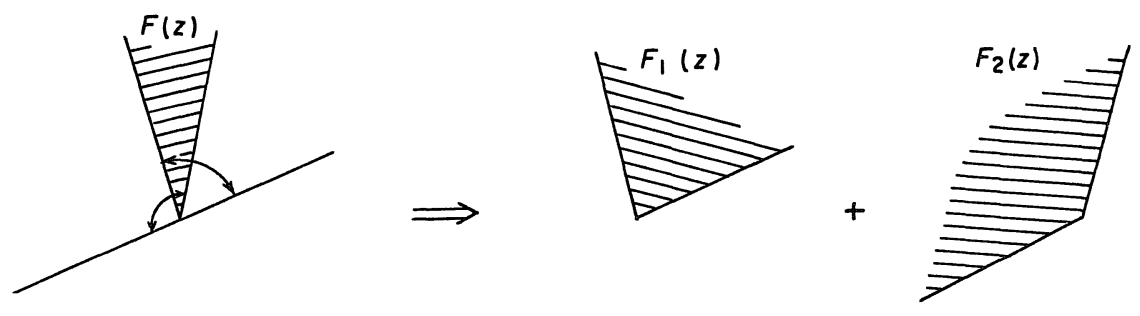

Fig. 1.67

Another way to understand the "singular spectrum" is the Fourier transform, and micro-analyticity and macro-causality in physics are connected by the Fourier transform. Here we do not enter into this subject.

\section{§ 2. Micro-differential Operators and Holonomic Systems}

In $\S 1$ the sheaf $\mathscr{B}$ of hyperfunctions was introduced naturally through the study of differential equations and the sheaf $\mathscr{C}$ of microfunctions was introduced through the study of the structure of manifolds. The third step is to generalize the concept of differential operators so as to fit with $\mathscr{C}$ and $\sqrt{-1} S^{*} M$.

Let us consider a differential operator $D_{1}=\partial / \partial x_{1}$. A solution of the equation

$$
D_{1} f(x)=g(x)
$$

always exists but is not unique. Its ambiguity is a hyperfunction $h\left(x_{2}\right.$, $\cdots, x_{n}$ ) independent of $x_{1}$. It is easy to see that

$$
\text { S.S.h } \subset\left\{(x, i \infty \xi) \in \sqrt{-1} S^{*} M \mid \xi_{1}=0\right\} .
$$




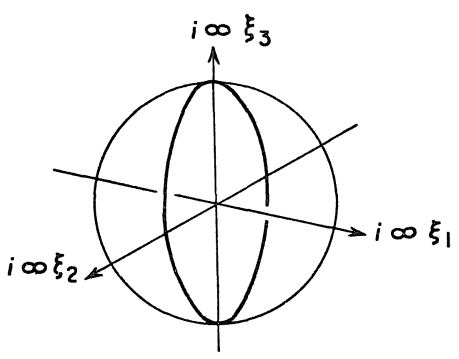

Fig. 2.3 S.S. $h\left(x_{2}, x_{3}\right)$

Therefore if we consider $(2 \cdot 1)$ microlocally in the neighborhood of a point $(x, i \infty \xi)$ in $\sqrt{-1} S^{*} M$ with $\xi_{1} \neq 0$, the microfunction solution is unique. In other words, $D_{1}^{-1}$ is well defined micro-locally on $\sqrt{-1} S^{*} M$ if $\xi_{1} \neq 0$.

Definition 2. 4. Let $\left(x_{0}, \infty \xi_{0}\right)$ be a point of $P^{*} X^{*}$, and $P_{j}(x, \xi)$ $(-\infty<j \leqq m)$ be a holomorphic function defined at $\left(x_{0}, \xi_{0}\right)$ and homogeneous of degree $j$ with respect to $\xi$. We require that

$$
\sqrt[-j]{\left|P_{j}(x, \xi)\right| /(-j) !} \quad(-\infty<j \leqq m)
$$

are locally uniformly bounded. Then the formal expression

$$
P(x, D)=\sum_{j=-\infty}^{m} P_{j}(x, D)
$$

represents a microdifferential operator defined at $\left(x_{0}, \infty \xi_{0}\right)$. Microdifferential operators defined at $\left(x_{0}, \infty \xi_{0}\right)$ form a non commutative ring $\mathcal{E}_{\left(x_{0}, \infty \xi_{0}\right)}$.

Through the two-fold embedding

$$
\sqrt{-1} S^{*} M \rightarrow P^{*} X
$$

we consider $P^{*} X$ as a complexification of $\sqrt{-1} S^{*} M$. Then $\mathcal{E}_{(x, i \infty \xi)}$ operates on $\mathscr{C}_{(x, i \infty \xi)}$.

Example 2.8. Let $P(x, D)=\sum_{j=-\infty}^{0} a_{j}(x)(-j) ! D^{j}$.

$$
P(x, D) \theta(x)=\sum_{j=-\infty}^{0} a_{j}(x) x_{+}^{-j} .
$$

$* P^{*} X=\left(T^{*} X-X\right) /(\boldsymbol{C}-\{0\})($ See $(1.50)$.$) .$ 
Then at $(0, i \infty d x)=\left(\sum_{j=-\infty}^{0} a_{j}(x) x^{-j}\right) \theta(x)$ where $\sum_{j=-\infty}^{0} a_{j}(x) x^{-j}$ is convergent because of $(2 \cdot 5)$. Thus a microdifferential operator can be replaced by a multiplication of a holomorphic function in this case.

Definition 2.9. We call $P_{m}(x, \xi)$ in the expression $(2 \cdot 6)$ the principal symbol of $P(x, D)$. We denote it by $\sigma(P)$. It has an intrinsic meaning as a homogeneous holomorphic function on $T^{*} X$ independent of coordinate transformations.

$$
\begin{aligned}
& \sigma(P \cdot Q)=\sigma(P) \sigma(Q) \\
& \sigma([P, Q])=\{\sigma(P), \sigma(Q)\}
\end{aligned}
$$

where $\{$,$\} means the Poisson bracket$

$$
\{f, g\}=\sum_{j=1}^{n}\left(\frac{\partial f}{\partial \xi_{j}} \frac{\partial g}{\partial x_{j}}-\frac{\partial f}{\partial x_{j}} \frac{\partial g}{\partial \xi_{j}}\right) .
$$

Through out this section and $\S 4$, we shall see how "commutative" principal symbols control "non-commutative" micro-differential operators.

\section{Theorem 2.13.}

If $\sigma(P)\left(x_{0}, \infty \xi_{0}\right) \neq 0, P(x, D)$ has a unique inverse in $\mathcal{E}_{\left(x_{0}, \infty \xi_{0}\right)}$. We call $P(x, D)$ elliptic.

Corollary 2.14. (Sato's fundamental theorem) If a microfunction $f(x)$ satisfies a micro-differential equation

$$
P(x, D) f(x)=0,
$$

then we have

$$
S . S . f(x) \subset\left\{(x, i \infty \xi) \in \sqrt{-1} S^{*} M \mid \sigma(P)(x, i \xi)=0\right\} .
$$

Thus a system of microdifferential equations enables us to estimate the singular spectrum of a microfunction which satisfies the system.

Exercise 2.17. Prove Weyl's lemma from $(2 \cdot 16)$ and $(1 \cdot 56)$. Weyl's lemma: a solution of an elliptic linear differential equation $P u=0$ is real analytic. 
Exercise 2.18. Determine $S . S . \delta\left(x_{1}\right) \cdots \delta\left(x_{r}\right)(1 \leqq r \leqq n)$ by the above method.

Let us consider a system of micro-differential equations

$$
P_{1} u=0, \cdots, P_{N} u=0 \text {. }
$$

A solution of $(2 \cdot 15)$ satisfies

$$
Q u=0 \quad \text { for } Q=R_{1} P_{1}+\cdots+R_{N} P_{N} \text {. }
$$

But $\sigma\left(P_{1}\right)(x, i \xi)=0, \cdots, \sigma\left(P_{N}\right)(x, i \xi)=0$ do not always imply $\sigma(Q)(x$, $i \xi)=0$. Thus we require a more intrinsic definition of what is a system of micro-differential equations.

Definition 2.21. A system of micro-differential equations is a coherent left $\mathcal{E}$-Module. This means that a system $\mathcal{M}$ has a representation (not unique)

$$
\begin{aligned}
& 0 \longleftarrow \mathscr{M} \longleftarrow \varepsilon^{s} \longleftarrow \varepsilon^{t} \\
& P_{1} u_{1}+\cdots+P_{s} u_{s} \leftrightarrow\left(P_{1}, \cdots, P_{s}\right) \\
& \left(\sum_{j} R_{j} P_{j 1}, \cdots, \sum_{j} R_{j} P_{j s}\right) \leftrightarrow\left(R_{1}, \cdots, R_{t}\right)
\end{aligned}
$$

where $u_{1}, \cdots, u_{s} \in \mathcal{M}$ and $\left(P_{j k}\right)_{\substack{j=1, \ldots, t \\ k=1, \cdots, s}}$ is a matrix of micro-differential operators. This representation corresponds to a usual expression

$$
\left.\sum_{k=1}^{s} P_{j k} u_{k}=0 \quad(j=1, \cdots, t) . *\right)
$$

Exercise 2.24. Show that differential equations

$$
(x D-\lambda) u=0
$$

and

$$
(x D-\lambda+1) v=0
$$

are different representations of the same differential equation if and only

* $\operatorname{Ham}_{\varepsilon}(\mathscr{M}, \mathcal{C})$ represents the sheaf of microfunction solutions of $\mathscr{M}$. The image of a set of generators $\left(u_{1}, \cdots, u_{s}\right)$ by an $\mathcal{E}$-homomorphism from $\mathcal{M}$ to $\mathcal{C}$ represents a solution of (2.23). 
if $\lambda \neq 0$. If we consider $(2 \cdot 25)$ and $(2 \cdot 26)$ as a micro-differential equation at $(0, i \infty \xi)(\xi \neq 0)$ they represent the same equation.

Definition 2.27. The characteristic variety of $\mathscr{M}$ is the support of $\mathcal{M}$ as a sheaf on $P^{*} X$. We denote it by S.S. $\mathcal{M}$.

From now on we assume that $s=1$ and we fix a generator $u$ and a coherent left ideal $g$ such that

$$
\mathscr{H}=\varepsilon u=\varepsilon / g .
$$

This means

$$
P u=0 \quad \text { for } P \in g \text {. }
$$

Then we have

$$
S . S . \mathscr{M}=\left\{(x, \infty \xi) \in P^{*} X \mid \sigma(P)(x, \xi)=0 \text { for } \forall P \in g\right\} .
$$

Exercise 2.31. Prove (2.30) using Theorem (2.13).

Let $V$ be a subvariety in $T^{*} X . \quad V$ is called involutory when

$$
f \equiv g \equiv 0 \text { on } V \text { implies }\{f, g\} \equiv 0 \text { on } V \text {, }
$$

and isotropic when

$$
\left.\sum_{j=1}^{n} d \xi_{j} \wedge d x_{j}\right|_{V} \equiv 0
$$

If $V$ is conic, this is equivalent to

$$
\left.\sum_{j=1}^{n} \xi_{j} d x_{j}\right|_{V} \equiv 0
$$

A hypersurface in $T^{*} X$ is involutory. A submanifold defined by $x_{1}=0$ and $\xi_{1}=0$ is not involutory. A submanifold defined by $x_{1}=\cdots=x_{r}$ $=\xi_{r+1}=\cdots=\xi_{n}=0$ is involutory and isotropic.

The dimension of an involutory subvariety is equal to or more than $n$, and the dimension of an isotropic subvariety is equal to or less than $n$.

We identify a conic (in $\xi$ ) subvariety (other than the zero section $X$ ) in $T^{*} X$ with a subvariety in $P^{*} X$. 
Theorem 2.35. (Fundamental theorem in the theory of linear differential equations). The characteristic variety of a system is involutory.

Corollary 2.36. $\operatorname{dim}$ S.S. $\mathscr{M} \geqq n$.

Exercise 2.37. Prove (2.35) under the assumption that

$$
J=\text { the ideal in } \mathcal{O}_{T^{*} X} \text { generated by }\{\sigma(P) \mid P \in g\}
$$

is reduced, that is, $f(x, \xi) \in J$ if and only if $f(x, \xi) \equiv 0$ on S.S.M.M. (Hint. Use $(2 \cdot 11)$.)

Definition 2.39. An involutory subvariety with $n$ dimensions or equivalent to say an isotropic subvariety with $n$ dimensions is called holonomic. A system $\mathscr{M}$ is called holonomic if S.S. $\mathscr{M}$ is holonomic.

Theorem 2.40. The solution space of a linear differential holonomic system is finite dimensional.

A function with a natural origin maybe satisfies a holonomic system. "Study functions microlocally through holonomic systems." This is the principle of MICROLOCAL CALCULUS and Theorem $(2 \cdot 40)$ gives a basis to our principle. We call solutions of a holonomic system holonomic hyper-(micro-) functions.

Let $Y$ be an irreducible subvariety in $X$. If $Y$ is non-singular the conormal bundle $T_{Y}{ }^{*} X$ can be defined as (1.48). In general we define $T_{Y}{ }^{*} X$ to be the Zariski closure in $T^{*} X$ of the conormal bundle of the non singular part of $Y$.

Theorem 2.41. $T_{Y}{ }^{*} X$ is an irreducible conic holonomic subvariety. Conversely if $\Lambda$ is an irreducible conic holonomic subvariety, we have

$$
\Lambda=T_{Y}^{*} X
$$

where $Y=\pi(\Lambda)$. 
Exercise 2.43. Prove theorem $(2 \cdot 41)$. It is sufficient to prove it when $Y=\pi(\Lambda)$ is a nonsigular submanifold.

Let $\Omega_{X}^{n}$ be the sheaf of $n$ forms on $X$. We can define a sheaf of $($ micro $=)$ differential operators which operate on $\sqrt{\Omega_{X}^{n}}$.

$$
\widetilde{\mathscr{D}}_{X}=\sqrt{\Omega_{X}^{n}} \otimes \mathscr{D}_{X} \otimes{\sqrt{\Omega_{X}^{n}}}^{-1}
$$

and

$$
\widetilde{\mathcal{E}}_{X}=\sqrt{\Omega_{X}^{n}} \otimes \mathcal{E}_{X} \otimes{\sqrt{\Omega_{X}^{n}}}^{-1}
$$

This means that $\sqrt{d x} P\left(x, D_{x}\right) \sqrt{d x}{ }^{-1}$ and $\sqrt{d y} Q\left(y, D_{y}\right) \sqrt{d y^{-1}}$ are equal if and only if

$$
\sqrt{\frac{d x}{d y}} P\left(x, D_{x}\right) \sqrt{\frac{d x}{d y}}^{-1}=Q\left(y, D_{y}\right)
$$

where $x$ and $y$ denote two local coordinates. In this sheaf not only principal symbol but also the following next symbol is invariant.

$$
P_{m-1}(x, \xi)-\frac{1}{2} \sum_{j=1}^{n} \frac{\partial^{2} P_{m}}{\partial x_{j} \partial \xi_{j}}(x, \xi) .
$$

Exercise 2.48. Check in some simple cases (2.47) is invariant under the coordinate transformation.

Then it is easy to see that for $P(x, D) \in \mathscr{D}_{X}$ or $\mathcal{E}_{X}, \widetilde{L}_{P}=\sqrt{d x}{ }^{-1} L_{p} \sqrt{d x}$ is a first order linear differential operator defined in $T^{*} X$ independent of local coordinates, where

$$
L_{P}=H_{P_{m}}+P_{m-1}(x, \xi)-\frac{1}{2} \sum_{j=1}^{n} \frac{\partial^{2} P_{m}}{\partial x_{j} \partial \xi_{j}} *
$$

Let $\mathscr{M}=\mathcal{E} u=\mathcal{E} / \mathrm{g}$ be a holonomic system whose support is a nonsingular holonomic manifold $\Lambda$. Then for $P(x, D) \in \mathcal{g}, L_{P}$ is a differential operator on $\Lambda$ and the system of differential equations on $\Lambda$

$$
\widetilde{L}_{P} s=0, \quad P(x, D) \in g
$$

\footnotetext{
* $H_{P_{m}}(f)=\left\{P_{m}, f\right\}$.
} 
where $\left.s \in \sqrt{\Omega_{\Lambda}^{n}} \otimes{\sqrt{\Omega_{X}^{n}}}^{-1} *\right)$ has finite dimensional solutions. Here $H_{P_{m}}$ operates on $\sqrt{\Omega_{\Lambda}^{n}}$ as the Lie derivative $L_{H_{P_{m}}} * *$ ).

From now on we assume that $\mathscr{M}$ is simple on $\mathcal{A}$, that is, $J$ in $(2 \cdot 38)$ is reduced. Then $(2 \cdot 50)$ has a unique (up to constant multiple) analytic solution on $\Lambda$. We denote it by $\sigma_{A}(u)$ and call it the principal symbol of the holonomic system $\mathcal{E} u=\varepsilon / g$.

\section{Definition 2.51.}

$\sigma_{\Lambda}(u)$ is homogeneous in $\xi$ and we call its homogeneous degree the order and denote it by $\left.\operatorname{ord}_{\Lambda}(u) . * * *\right)$

Example 2.52.

$$
\begin{aligned}
& g=\mathscr{D} x_{1}+\cdots+\mathscr{D} x_{r}+\mathscr{D} D_{r+1}+\cdots+\mathscr{D} D_{n}, \\
& \Lambda=\left\{(x, \xi) \mid x_{1}=\cdots=x_{r}=\xi_{r+1}=\cdots=\xi_{n}=0\right\}, \\
& \sigma_{\Lambda}(u)=\sqrt{\frac{d \xi_{1} \cdots d \xi_{r} d x_{r+1} \cdots d x_{n}}{d x_{1} \cdots d x_{r} d x_{r+1} \cdots d x_{n}}}, \\
& \operatorname{ord}_{\Lambda}(u)=\frac{r}{2} .
\end{aligned}
$$

Example 2.53.

$$
\begin{aligned}
& g=\mathscr{D}\left(x_{1} D_{1}-\alpha\right)+\mathscr{D}\left(x_{2} D_{2}-\beta\right)+\mathscr{D} D_{3}+\cdots+\mathscr{D} D_{n}, \\
& \Lambda_{1}=\left\{x_{1}=x_{2}=\xi_{3}=\cdots=\xi_{n}=0\right\}, \\
& \Lambda_{2}=\left\{x_{1}=\xi_{2}=\xi_{3}=\cdots=\xi_{n}=0\right\} \\
& \sigma_{\Lambda_{1}}(u)=\xi_{1}^{-\alpha-1} \xi_{2}^{-\beta-1} \sqrt{\frac{d \xi_{1} d \xi_{2} d x_{3} \cdots d x_{n}}{d x_{1} d x_{2} d x_{3} \cdots d x_{n}}}
\end{aligned}
$$

${ }^{*} s$ is well-defined up to constant multiple. If $\Lambda$ and $M$ are real, $s$ is defined without ambiguity as a section of $\sqrt{\mathcal{C}_{1}} \otimes \sqrt{\mathcal{C V}_{M}{ }^{-1}}$ where $C$ denotes the sheaf of volume elements.

** $L_{\sum_{i=1}^{n} a_{i}(x) \frac{\partial}{\partial x_{i}}} f(x) \sqrt{d x_{1 \wedge \cdots \wedge} d x_{u}}=\left\{\sum_{i=1}^{n} a_{i}(x) \frac{\partial f}{\partial x_{i}}(x)+\frac{1}{2} \sum_{i=1}^{n}(-)^{i-1} \frac{\partial a_{i}}{\partial x_{i}} f(x)\right\} \sqrt{d x_{1 \wedge \cdots \wedge} d x_{n}}$.

*** Note that 'principal symbol' and 'order' are defined only if we fix a generator of M. An exchange of generators causes a difference of an integer in orders. See Exercise (2.24). 


$$
\begin{aligned}
& \operatorname{ord}_{\Lambda_{1}}(u)=-\alpha-\beta-1 \\
& \sigma_{\Lambda_{2}}(u)=\xi_{1}{ }^{-\alpha-1} x_{2}{ }^{\beta} \sqrt{\frac{d \xi_{1} d x_{2} d x_{3} \cdots d x_{n}}{d x_{1} d x_{2} d x_{3} \cdots d x_{n}}} \\
& \operatorname{ord}_{\Lambda_{2}}(u)=-\alpha-\frac{1}{2}
\end{aligned}
$$

Example 2.54.

$$
\begin{aligned}
& g=\mathscr{D}\left(\frac{1}{2} x_{1} D_{1}+x_{2} D_{2}+\alpha\right)+\mathscr{D}\left\{\frac{1}{2}\left(\frac{1}{2} D_{1}+x_{1} D_{2}\right) D_{1}+\beta D_{2}\right\} \\
& \Lambda_{1}=\left\{\xi_{1}=x_{2}=0\right\} \\
& \Lambda_{2}=\left\{x_{2}=x_{1}^{2}, \xi_{1}+2 x_{1} \xi_{2}=0\right\} \\
& \sigma_{\Lambda_{1}}(u)=x_{1}^{-2 \beta} \xi_{2}^{\alpha-\beta-1} \sqrt{\frac{d x_{1} d \xi_{2}}{d x_{1} d x_{2}}}, \\
& \operatorname{ord}_{\Lambda_{1}}(u)=\alpha-\beta-\frac{1}{2}, \\
& \sigma_{\Lambda_{2}}(u)=x_{1}^{2 \beta-1} \xi_{2}^{\alpha+\beta-3 / 2} \sqrt{\frac{d x_{1} d \xi_{2}}{d x_{1} d x_{2}}} \\
& \operatorname{ord}_{\Lambda_{2}}(u)=\alpha+\beta-1 .
\end{aligned}
$$

To study singular points of a characteristic variety only a singularity of codimension 1 (that is of dimensions $n-1$ ) plays an essential role. In this sense it is important to determine if two (or more) irreducible components of the characteristic variety have a codimension 1 intersection or not. The structure of a holonomic system is visualized by 'holonomy diagram' which represents the configuration of irreducible components of the characteristic variety.

\section{$\S 3$. Several Operations on Functions and Systems}

In this section we show by an example how the Feynman amplitude can be defined as a hyperfunction. In the course we explain several operations (substitution, integration, product and compactification).

Consider the following Feynman graph (Fig. 3.1). 


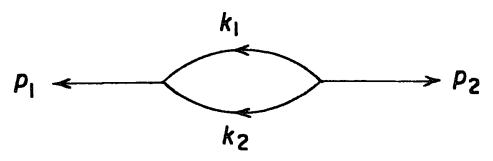

Fig. 3.1

The Feynman amplitude with parameter $\lambda_{2}$ of this graph is an integral of the form

$$
\begin{gathered}
\iint \delta\left(p_{1}-k_{1}-k_{2}\right) \delta\left(p_{2}+k_{1}+k_{2}\right) \frac{1}{\left(k_{1}{ }^{2}-m_{1}{ }^{2}+i 0\right)^{\lambda_{1}}} \\
\left.\times \frac{1}{\left(k_{2}{ }^{2}-m_{2}{ }^{2}+i 0\right)^{\lambda_{2}}} d k_{1} d k_{2} \cdot *\right)
\end{gathered}
$$

To define $(3 \cdot 2)$ as a hyperfunction we prepare several operations.

Let $f: N \rightarrow M, y \mapsto x=f(y)$ be a real analytic map. We have the following diagram

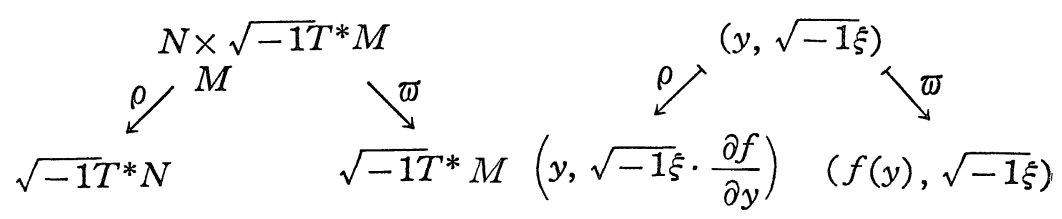

We define $T_{N}^{*} M$ by an exact sequence

$$
0 \rightarrow T_{N}^{*} M \rightarrow \underset{M}{\times} T^{*} M \rightarrow T^{*} N \rightarrow 0 .
$$

In a local coordinate

$$
\sum_{i=1}^{n} \xi_{i} d x_{i} \in T_{N}^{*} M
$$

if and only if $\sum_{i=1}^{n} \xi_{i} \frac{\partial f_{i}}{\partial y_{j}}=0$ for any $j$.

We shall not only identify the zero section of $\sqrt{-1} T^{*} M$ with $M$ but also identify a conic set of $\sqrt{-1} T^{*} M-M$ with the corresponding set in $\sqrt{-1} S^{*} M$. We consider a hyperfunction $u(x)$ as a microfunction defined all over $\sqrt{-1} T^{*} M$. Therefore it is real analytic if and only if S.S.u $\left.(x) \subset M^{* *}\right)$

(i) Substitution.

Let $u(x)$ be a microfunction on $\sqrt{-1} T^{*} M . \quad u(f(y))$ is defined as

$* p_{j}$ and $k_{l}$ are $\nu$ dimensional Minkowski vectors.

** We consider the relations between $T^{*} X$ and $P^{*} X$, and $\mathscr{D}_{X}$ and $\mathcal{E}_{X}$ likewise. 
a microfunction at $(y, \sqrt{-1} \eta) \in \sqrt{-1} T^{*} N$ if

$$
\rho: \varpi^{-1}(S . S . u(x)) \rightarrow \sqrt{-1} T^{*} N
$$

is proper in a neighborhood of $(y, \sqrt{-1} \eta)$. For a hyperfunction this is equivalent to

$$
\left.\varpi^{-1}(S . S . u(x)) \cap \sqrt{-1} T_{N}^{*} M=N^{*}\right) .
$$

We can estimate $S . S . u(f(y))$.

$$
\text { S.S.u }(f(y)) \subset \rho\left(\varpi^{-1}(S . S . u(x))\right) .
$$

(ii) Integration.

Let $v(y) d y$ be a microfunction density on $\sqrt{-1} T^{*} N$. $\int_{f^{-1}(x)} v(y) d y$ is defined as a microfunction at $(x, \sqrt{-1} \xi) \in \sqrt{-1} T^{*} M$ if

$$
\varpi: \rho^{-1}(S . S . v(y) d y) \rightarrow \sqrt{-1} T^{*} M
$$

is proper. We can estimate S.S. $\int_{f^{-1}(x)} v(y) d y$.

$$
S . S .\left(\int_{f^{-1}(x)} v(y) d y\right) \subset \varpi\left(\rho^{-1}(S . S . v(y) d y)\right) \text {. }
$$

(iii) Product.

Let $u_{1}(x)$ and $u_{2}(x)$ be hyperfunctions on $M . u_{1}(x) \cdot u_{2}(x)$ is defined as a hyperfunction on $M$ if

$$
\text { S.S. } u_{1}(x) \cap S . S .{ }^{a} u_{2}(x) \subset M
$$

where $S . S .^{a} u_{2}(x)=\left\{(x, \sqrt{-1} \xi) \in \sqrt{-1} T^{*} M \mid(x,-\sqrt{-1} \xi) \in S . S . u_{2}(x)\right\}$. We can estimate $S . S .\left(u_{1}(x) u_{2}(x)\right)$.

$$
\begin{aligned}
\text { S.S. }\left(u_{1}(x) u_{2}(x)\right) \subset & \left\{\left.(x, i \xi) \in \sqrt{-1} T^{*} M\right|^{\exists}\left(x, i \xi_{l}\right) \in S . S . u_{l}(x)\right. \\
& \left.(l=1,2) \text { such that } \xi_{1}+\xi_{2}=\xi\right\} .
\end{aligned}
$$

We show how to apply these formulas (i) (iii) to define $(3 \cdot 2)$ and estimate its singular spectrum.

First we define the integrand $\Phi_{G}(p, k)$ as a hyperfunction on $N=\left\{\left(p_{1}\right.\right.$, $\left.\left.p_{2}, k_{1}, k_{2}\right)\right\}$. We denote by $x_{j}$ and $v_{l}$ the dual coordinates of $p_{j}$ and $k_{l}$

$* N$ means zero vectors. 
in $\sqrt{-1} T^{*} N . \quad(3 \cdot 6)$ is always satisfied if $f$ is a smooth map. ${ }^{*)}$ Therefore $\delta\left(p_{1}-k_{1}-k_{2}\right), \delta\left(p_{2}+k_{1}+k_{2}\right),\left(k_{1}{ }^{2}-m_{1}{ }^{2}+i 0\right)^{-\lambda_{1}}$ and $\left(k_{2}{ }^{2}-m_{2}{ }^{2}+i 0\right)^{-\lambda_{2}}$ are well-defined. Their singular spectra are given by

$$
\begin{aligned}
S . S . \delta\left(p_{1}-k_{1}-k_{2}\right)= & \left\{\left(p, k, \sqrt{-1} x_{1} d\left(p_{1}-k_{1}-k_{2}\right)\right) \in \sqrt{-1} T^{*} N \mid\right. \\
& \left.p_{1}-k_{1}-k_{2}=0\right\}, \\
S . S . \delta\left(p_{2}+k_{1}+k_{2}\right)= & \left\{\left(p, k, \sqrt{-1} x_{2}\left(p_{2}+k_{2}+k_{2}\right)\right) \in \sqrt{-1} T^{*} N \mid\right. \\
& \left.p_{2}+k_{1}+k_{2}=0\right\},
\end{aligned}
$$$$
\text { S.S. }\left(k_{1}{ }^{2}-m_{1}{ }^{2}+i 0\right)^{-\lambda_{1}}=\left\{\left(p, k, \sqrt{-1} \frac{\alpha_{1}}{2} d\left(k_{1}{ }^{2}-m_{1}{ }^{2}\right)\right) \in \sqrt{-1} T^{*} N \mid\right.
$$$$
\left.\alpha_{1} \geqq 0, \quad \alpha_{1}\left(k_{1}^{2}-m_{1}^{2}\right)=0\right\},
$$$$
S . S .\left(k_{2}{ }^{2}-m_{2}{ }^{2}+i 0\right)^{-\lambda_{2}}=\left\{\left(p, k, \sqrt{-1} \frac{\alpha_{2}}{2} d\left(k_{2}{ }^{2}-m_{2}{ }^{2}\right)\right) \in \sqrt{-1} T^{*} N \mid\right.
$$

$$
\left.\alpha_{2} \geqq 0, \quad \alpha_{2}\left(k_{2}^{2}-m_{2}^{2}\right)=0\right\} .
$$

Then using $(3 \cdot 12)$ we have

$$
\begin{aligned}
\text { S.S. } \Phi_{G}(p, k)= & \left\{(p, k, \sqrt{-1}(x, v)) \in \sqrt{-1} T^{*} N \mid\right. \\
& \exists \alpha_{l} \geqq 0 \text { such that } p_{1}-k_{1}-k_{2}=0, p_{2}+k_{1}+k_{2}=0, \\
& \alpha_{1}\left(k_{1}{ }^{2}-m_{1}{ }^{2}\right)=0, \alpha_{2}\left(k_{2}{ }^{2}-m_{2}{ }^{2}\right)=0, v_{1}+x_{1}-x_{2}-\alpha_{1} k_{1}=0, \\
& \left.v_{2}+x_{1}-x_{2}-\alpha_{2} k_{2}=0\right\} .
\end{aligned}
$$

If we apply $(3 \cdot 10) * *)$ we have

(3.15) S.S.F $F_{G}(p) \subset\left\{(p, \sqrt{-1} x) \in \sqrt{-1} T^{*} M \mid \exists k_{1}, k_{2}, \alpha_{1} \geqq 0\right.$,

$$
\begin{aligned}
& \alpha_{2} \geqq 0, p_{1}-k_{1}-k_{2}=0, p_{2}+k_{1}+k_{2}=0, \alpha_{l}\left(k_{l}{ }^{2}-m_{l}{ }^{2}\right)=0, \\
& \left.x_{1}-x_{2}=\alpha_{l} k_{l}(l=1,2)\right\} .
\end{aligned}
$$

These are the well-known Landau equations. There are two irreducible components. One on which $\alpha_{l} \not \equiv 0(l=1,2)$ is called the leading Landau singularity and is denoted by $\Lambda_{G}$.

The other is the conormal bundle of the submanifold $p_{1}+p_{2}=0$, on which $\alpha_{l} \equiv 0 \quad(l=1,2)$. We denote it by $\Lambda_{\text {point, }\{11,2\}}$ or $\Lambda_{p t}$ in short.

The condition (3.9) fails on $\Lambda_{p t}$. To overcome this difficulty we

* $f$ is smooth by difinition if $T_{N} * M=N$. See also the footnote on page 28 .

** Note that $\rho\left(N \times T^{*} M\right)=\left\{(p, k, \sqrt{-1}(x, 0)) \in \sqrt{-1} T^{*} N\right\}$ 
construct a compactification of $\left(k_{1}, k_{2}\right)$-space. We projectify each $\nu$ vector $k_{1}, k_{2}$.

$$
\left\{\left(p_{1}, p_{2},\left(k_{1}, s_{1}\right),\left(k_{2}, s_{2}\right)\right)\right\} \cong \boldsymbol{R}^{\nu} \times \boldsymbol{R}^{\nu} \times \boldsymbol{P}^{\nu} \times \boldsymbol{P}^{\nu} .
$$

Carrying out the integration of $\delta$ functions we can rewrite $(3 \cdot 2)$ as an integral over the space of loop momentum.

$$
N_{\text {loop }}=\left\{(p, k) \in N \mid p_{1}-k_{1}-k_{2}=p_{2}+k_{1}+k_{2}=0\right\} .
$$

The closure of $N_{\text {loop }}$ in $(3 \cdot 16)$ is defined by

$$
p_{1}-\frac{k_{1}}{s_{1}}-\frac{k_{2}}{s_{2}}=0 \text {. }
$$

Therefore it is non singular and as a local coordinate we may take $p_{1}$ and $\left(k_{1}, s_{1}\right)$. Abbreviating suffix 1 , we define the integrand at infinity as the form

$$
|s|^{2\left(\lambda_{1}+\lambda_{2}\right)-\nu-1}\left(k^{2}-s^{2} m_{1}^{2}+i 0\right)^{-\lambda_{1}}\left((k-s p)^{2}-s^{2} m_{2}^{2}+i 0\right)^{-\lambda_{2}} d(s, k) .
$$

This is a hyperfunction density along the fiber with parameters $\lambda_{1}, \lambda_{2}$. It is mermorphic in $\lambda_{1}, \lambda_{2}$ with simple poles at

$$
\lambda_{1}+\lambda_{2}-\frac{\nu}{2}=0,-1, \cdots
$$

Exercise 3.21. Examine the well-definedness of $(3 \cdot 19)$. (Hint. $+i 0$ plays a role.)

Exerise $3 \cdot 22$. Show that the singular spectrum of the integral of $(3 \cdot 19)$ is contained in $\Lambda_{G} \cup \Lambda_{p t}$.

We have explained how to define a hyperfunction and estimate its singular spectrum. Further we shall explain how to construct the system which it satisfies and how to estimate its characteristic variety.

We define the formal adjoint $P^{*}\left(x^{\prime}, D_{x^{\prime}}\right)$ of a microdifferential operator $P\left(x, D_{x}\right)$ by

$$
P(x, D) \delta\left(x-x^{\prime}\right)=P^{*}\left(x^{\prime}, D_{x^{\prime}}\right) \delta\left(x-x^{\prime}\right) .
$$

Then $\varepsilon_{X}$ operates on $\varepsilon_{X} \otimes \Omega_{X}^{n}$ from the right by

$$
(Q(x, D) d x) P(x, D)=P^{*}(x, D) Q(x, D) d x
$$


We define $\mathcal{E}_{Y_{\rightarrow X}^{f} X}$ to be the following holonomic system tensored by $\Omega_{X}^{n}$ from the right.

$$
\begin{aligned}
& \mathcal{E}_{Y<X} \delta(x-f(y)) \otimes \Omega_{X}^{n} \\
& \quad=\mathcal{E}_{Y \times X} /\left(\sum_{i=1}^{n} \mathcal{E}_{Y \times X}\left(x_{i}-f_{i}(y)\right)+\sum_{j=1}^{m} \varepsilon_{Y \times X}\left(D_{y_{j}}-\sum_{i=1}^{n} \frac{\partial f_{i}}{\partial y_{j}} D_{x_{i}}\right) \otimes \Omega_{X}^{n}\right) .
\end{aligned}
$$

We denote the generator $\delta(x-f(y)) \otimes d x$ by $1_{Y \rightarrow X}$.

The characteristic variety of $\mathcal{E}_{Y \rightarrow X}$ is the conormal bundle of the graph $\Gamma$ of $f: Y \rightarrow X$. We identify it with $Y \times T^{*} X$.

$$
T_{\Gamma}^{*}(Y \times X) \cong Y \times T^{*} X
$$

$\mathcal{E}_{Y \rightarrow X}$ is a (left $\mathcal{E}_{Y}$, right $\left.\varepsilon_{X}\right)$-Module.

Several operations on systems.

(i) Substitution.

Let $\mathscr{M}_{X}$ be a system on $T^{*} X$. The induced system

$$
\mathscr{M}_{Y}=f^{*} \mathscr{M}_{X}=\rho_{*}\left(\varepsilon_{Y \rightarrow X} \bigotimes_{X} \mathscr{M}_{X}\right)
$$

is defined as a system at $(y, \eta) \in T^{*} Y$ if

$$
\rho: \varpi^{-1}\left(S . S . \mathscr{M}_{X}\right) \rightarrow T^{*} Y
$$

is proper (hence with finite fibers) in a neighborhood of $(y, \eta)$. If $(3 \cdot 28)$ holds we say $\mathscr{M}_{X}$ is non-characteristic with respect to $f$. We can estimate S.S. $\mathscr{M}_{Y}$.

$$
\text { S.S. } \mathscr{M}_{Y} \subset \rho\left(\varpi^{-1}\left(S . S . \mathcal{M}_{X}\right)\right) \text {. }
$$

If $\mathscr{M}_{X}$ is holonomic, $\mathscr{M}_{Y}$ is holonomic. If $\mathscr{M}_{X}$ is simple holonomic with a generator $u$ and $\varpi$ is transversal to $\Lambda=S . S . \mathcal{M}_{X}$ and $\left.\rho\right|_{\varpi^{-1}(\Lambda)}$ is an embedding then $f^{*} u=1_{Y \rightarrow X} \otimes u \in \mathcal{M}_{Y}$ generates a simple holonomic system and

$$
\operatorname{ord}_{\rho\left(\varpi^{-1} \Lambda\right)}\left(f^{*} u\right)=\operatorname{ord}_{\Lambda}(u)
$$

Exercise 3.31. If $Y$ is a submanifold of codimension 1 in $X$ and $\mathscr{D}_{X}=\mathscr{D}_{X} / \mathscr{D}_{X} P(x, D)$, what is the condition $(3 \cdot 32)$ and what is $\mathscr{M}_{Y}$ ? 
Exercise 3.32. If $Y=\left\{x_{1}=\cdots=x_{d}=0\right\} \subseteq X=\left\{\left(x_{1}, \cdots, x_{d}, y_{1}, \cdots\right.\right.$, $\left.\left.y_{n-d}\right)\right\}$ and $\mathcal{M}_{X}=\mathcal{E}_{X} u=\mathcal{E}_{X} / g_{X}$, then the ideal which annihilates $1_{Y \rightarrow X} \otimes u$ consists of $P\left(y, D_{y}\right)$ such that $P\left(y, D_{y}\right)+\sum_{i=1}^{d} x_{i} R_{i}\left(x, y, D_{x}, D_{y}\right) \in g_{X}$.

(ii) Integration

Let $\mathscr{M}_{Y}$ be a right coherent Module on $T^{*} Y$. The induced system

$$
f_{*} \mathscr{M}_{Y}=\varpi_{*}\left(\mathscr{M}_{Y} \otimes \mathcal{E}_{\varepsilon_{Y}}\right)
$$

is defined as a right coherent Module at $(x, \xi) \in T^{*} X$ if

$$
\varpi: \rho^{-1}\left(S . S . \mathcal{M}_{Y}\right) \rightarrow T^{*} X
$$

is proper and with finite fibers. We can estimate $S . S . f_{*} \mathscr{M}_{Y}$.

$$
\text { S.S.f. } \mathscr{M}_{Y} \subset \varpi\left(\rho^{-1}\left(\text { S.S. } \mathscr{M}_{Y}\right)\right) \text {. }
$$

If $\mathscr{M}_{Y}$ is holonomic, $f_{*} \mathscr{M}_{Y}$ is holonomic. If $\mathscr{M}_{Y}$ is simple holonomic with a generator $u$ and $\rho$ is transversal to $\Lambda=S . S . \mathscr{M}_{Y}$ and $\left.\varpi\right|_{\rho^{-1}(\Lambda)}$ is an embedding, then $f_{*} u=u \otimes 1_{Y \rightarrow X} \in f_{*} \mathcal{M}_{Y}$ generates a simple holonomic right Module and

$$
\begin{aligned}
& \operatorname{ord}_{\Lambda}\left(d y^{-1} \otimes u\right)-\frac{1}{2} \operatorname{dim} Y \\
& \quad=\operatorname{ord}_{\rho^{-1}(\Lambda)}\left(d x^{-1} \otimes f_{*} u\right)-\frac{1}{2} \operatorname{dim} X .
\end{aligned}
$$
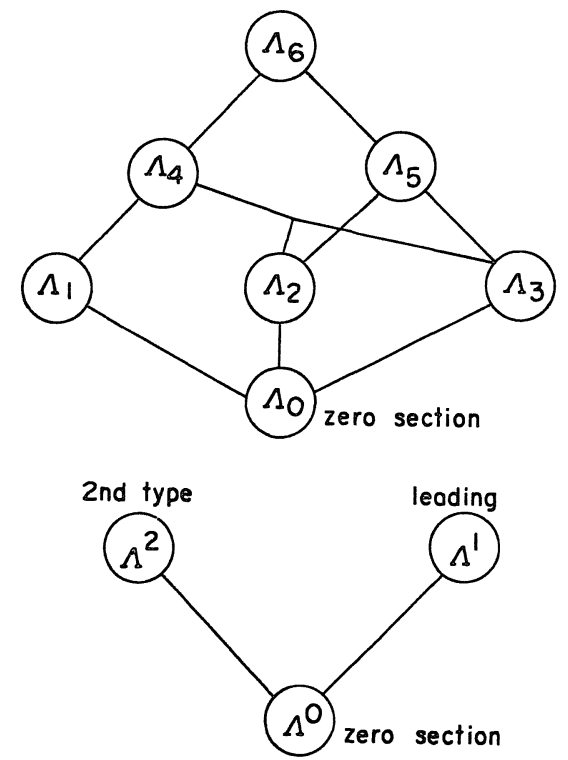

$$
\begin{array}{rl}
\pi\left(\Lambda_{i}\right) & (i=1, \cdots, 6) \\
i=1 & t=0 \\
2 & k^{2}-t^{2} m_{2}{ }^{2}=0 \\
3 & (t p-k)^{2}-t^{2} m_{1}{ }^{2}=0 \\
4 & t=k^{2}=0 \\
5 & k^{2}-t^{2} m_{2}{ }^{2}=0 \\
& t p^{2}-2 p \cdot k+t\left(m_{2}{ }^{2}-m_{1}{ }^{2}\right)=0 \\
6 & t=k^{2}=p \cdot k=0
\end{array}
$$

$$
\begin{aligned}
\pi\left(\Lambda^{i}\right) & (i=1,2) \\
i=1 & p^{2}-\left(m_{1}+m_{2}\right)^{2}=0 \\
2 & p^{2}=0
\end{aligned}
$$

Each segment represents an intersection of codimension 1 .

Fig. 3. 36 The holonomy diagram of a self energy graph. 
Exercise 3.35. If $f$ is a projection $Y=\{(x, y)\} \rightarrow X=\{x\}$ and $\mathcal{M}_{Y}$ $\varepsilon_{Y} u=\varepsilon_{Y} / g_{Y}$, then the ideal which annihilates $u \otimes 1_{Y \rightarrow X}$ consists of $P(x$, $\left.D_{x}\right)$ such that $P\left(x, D_{x}\right)+\sum_{i=1}^{m} D_{y_{i}} R_{i}\left(x, y, D_{x}, D_{y}\right) \in g_{Y}$. The condition $(3 \cdot 23)$ is slightly restrictive. It can be weakened for holonomic systems under some natural conditions so that the finiteness of the fiber is unnecessary. We give the holonomy diagram of the integrand and the integral of (3.2) after carrying out an integration of $\delta$ function. (Fig. 3.36)

\section{§4. Quantized Contact Transformation}

A contact transformation

$$
F: T^{*} X \rightarrow T^{*} Y
$$

is an isomorphism defined by

$$
\begin{aligned}
& \eta_{i}=f_{i}(x, \xi) \\
& y_{i}=g_{i}(x, \xi) \quad(i=1, \cdots, n),
\end{aligned}
$$

such that $f_{i}(x, \xi)$ is homogeneous of degree 1 with respect to $\xi$ and $g_{i}(x, \xi)$ of degree 0 and the canonical 1 form $\sum_{i=1}^{n} \eta_{i} d y_{i}$ is pulled back to $\sum_{i=1}^{n} \xi_{i} d x_{i}$

$$
\begin{aligned}
F^{*}\left(\sum_{i=1}^{n} \eta_{i} d y_{i}\right) & =\sum_{i=1}^{n} f_{i}(x, \xi) d g_{i}(x, \xi) \\
& =\sum_{i=1}^{n} \xi_{i} d x_{i} .
\end{aligned}
$$

There are two projections $\pi_{X}: T^{*}(X \times Y) \rightarrow T^{*} X$ and $\pi_{Y}: T^{*}(X \times Y) \rightarrow T^{*} Y$ defined by $\pi_{X}(x, y, \xi,-\eta)=(x, \xi)$ and $\pi_{Y}(x, y, \xi,-\eta)=(y, \eta)$.

Let us consider the graph $\Lambda$ of $F$.

$$
\begin{gathered}
\Lambda=\left\{(x, y, \xi,-\eta) \in T^{*}(X \times Y) \mid \eta_{i}=f_{i}(x, \xi),\right. \\
\left.y_{i}=g_{i}(x, \xi), \quad(i=1, \cdots, n)\right\} .
\end{gathered}
$$

$\Lambda$ is holonomic, and conversely if the graph of an isomorphism is holonomic it is a contact transformation.

Exercise 4.5. Check the above equivalence. 
A holonomic manifold is given by a generating function. We explain this in the case $\Lambda \subset T^{*} X=\left\{\left(x_{1}, \cdots, x_{n} ; \xi_{1}, \cdots, \xi_{n}\right)\right\}$.

We write $x^{\prime}=\left(x_{1}, \cdots, x_{d}\right), x^{\prime \prime}=\left(x_{d+1}, \cdots, x_{n}\right), \xi^{\prime}=\left(\xi_{1}, \cdots, \xi_{d}\right)$ and $\xi^{\prime \prime}$ $=\left(\xi_{d+1}, \cdots, \xi_{n}\right)$. A function of the form $F\left(x^{\prime}, \xi^{\prime \prime}\right)$ and homogeneous of degree 1 with respect to $\xi$ (we assume $d \neq n$ ) is called a generating function. $\Lambda$ defined by

$$
\Lambda=\left\{(x, \xi) \in T^{*} X \mid \xi^{\prime}=-\frac{\partial F}{\partial x^{\prime}}\left(x^{\prime}, \xi^{\prime \prime}\right), x^{\prime \prime}=\frac{\partial F}{\partial \xi^{\prime \prime}}\left(x^{\prime}, \xi^{\prime \prime}\right)\right\}
$$

is holonomic and any holonomic manifold is given likewise.

Exercise $4 \cdot 7$. Check the above equivalence.

\section{Example $4 \cdot 8$.}

Consider the icecream cone diagram (Fig. 4.8).

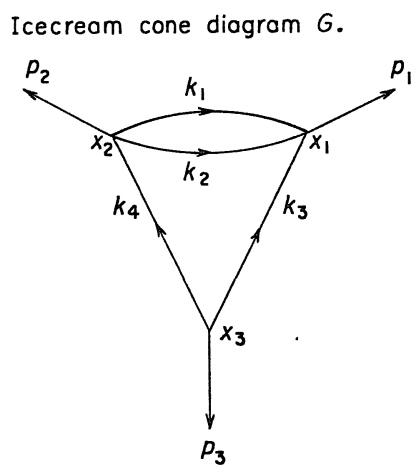

Fig. 4.8

The leading Landau singularity $\Lambda_{G}$ is given by Landau equations.

$$
\begin{aligned}
& p_{1}=k_{1}+k_{2}+k_{3}, \quad p_{2}=-k_{1}-k_{2}+k_{4}, \quad p_{3}=-k_{3}-k_{4}, \\
& k_{l}{ }^{2}=m_{l}{ }^{2} \quad(l=1,2,3), \\
& x_{1}-x_{2}=\alpha_{1} k_{1}=\alpha_{2} k_{2}, \quad x_{1}-x_{3}=\alpha_{3} k_{3}, \quad x_{2}-x_{3}=\alpha_{4} k_{4} .
\end{aligned}
$$

We can take a generating function

$$
\begin{aligned}
H(x)= & m_{1} \sqrt{\left(x_{1}-x_{2}\right)^{2}}+m_{2} \sqrt{\left(x_{1}-x_{2}\right)^{2}}+m_{3} \sqrt{\left(x_{1}-x_{3}\right)^{2}} \\
& +m_{4} \sqrt{\left(x_{2}-x_{3}\right)^{2}} .
\end{aligned}
$$


Exercise 4.11. Check $p=\frac{\partial H}{\partial x}$ is equivalent to $(4 \cdot 9)$

(Hint. $\quad k_{1}=\frac{m_{1}\left(x_{1}-x_{2}\right)}{\sqrt{\left(x_{1}-x_{2}\right)^{2}}}, \quad \alpha_{1}=\frac{\sqrt{\left(x_{1}-x_{2}\right)^{2}}}{m_{1}}$ etc.)

We call $H(x)$ a potential function. Consider a generating function

$$
\sum_{j=1}^{n} p_{j} \tilde{x}_{j}-\left(m_{3} \sqrt{\left(x_{1}-x_{3}\right)^{2}}+m_{4} \sqrt{\left(x_{2}-x_{3}\right)^{2}}\right) .
$$

It defines the following contact transformation. (Here we let $k_{3}=\frac{m_{3}\left(x_{1}-x_{3}\right)}{\sqrt{\left(x_{1}-x_{3}\right)^{2}}}$ and $k_{4}=\frac{m_{4}\left(x_{2}-x_{3}\right)}{\sqrt{\left(x_{2}-x_{3}\right)^{2}}}$.

$$
\begin{aligned}
& \widetilde{p}_{1}=p_{1}-k_{3}, \quad \widetilde{p}_{2}=p_{2}-k_{4}, \quad \widetilde{p}_{3}=p_{3}+k_{3}+k_{4}, \\
& \widetilde{x}_{j}=x_{j} \quad(j=1,2,3) .
\end{aligned}
$$

$(4 \cdot 12)$ reduces $(4 \cdot 9)$ to

$$
\begin{aligned}
& \widetilde{p}_{1}=k_{1}+k_{2}, \quad \tilde{p}_{2}=-k_{1}-k_{2}, \quad \widetilde{p}_{3}=0, \\
& k_{l}{ }^{2}=m_{l}{ }^{2} \quad(l=1,2), \\
& \widetilde{x}_{1}-\widetilde{x}_{2}=\alpha_{1} k_{1}=\alpha_{2} k_{2} .
\end{aligned}
$$

This is the leading Landau singularity $\Lambda_{G_{I_{0}-\{3,4\}}}$ of the subgraph $G_{I_{0}-\{3,4\}}$ obtained from $(4 \cdot 7)$ by deleting two lines $k_{3}$ and $k_{4}$. (Fig. 4.14)

The subgroph $G_{I_{0}}-\{3,4\}$. The contracted graph $G /\{1,2\}$.

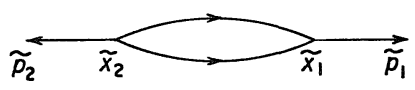

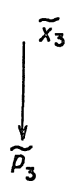

Fig. 4.14

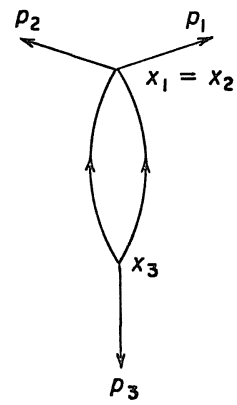

Fig. 4.15

Exercise $4 \cdot 15$. Into which the non-leading component $\Lambda_{G,\{1,2\}, \tau}$ (corresponding to the contracted graph $G /\{1,2\}$, Fig. (4-15)) is transformed 
by the above contact transformation? Show that $\Lambda_{G}$ and $\Lambda_{G /\{1,2\}, \tau}$ have a codimension 1 intersection.

Let $\Lambda \subset T^{*}(X \times Y)$ be the graph of a contact transformation $(4 \cdot 2)$ and $\mathscr{M}$ be a simple holonomic system with generator $K(x, y)$ with the characteristic variety $\Lambda$. On $\mathscr{M} \otimes \Omega_{X}^{n} \mathcal{E}_{X}$ operates from the left and $\mathcal{E}_{X}$ from the right.

The equation

$$
\begin{aligned}
P(x, & \left.D_{x}\right) K(x, y) d y \\
& =K(x, y) d y Q\left(y, D_{y}\right) \\
& =Q^{*}\left(y, D_{y}\right) K(x, y) d y
\end{aligned}
$$

gives an isomorphism between $\mathcal{E}_{X}$ and $\mathcal{E}_{Y}$. Because $\mathscr{M}$ is simple, there exist microdifferential equations of the forms

$$
\begin{aligned}
& \left\{D_{y_{i}}-f_{i}\left(x, D_{x}\right)+\text { lower order } K(x, y)=0\right. \\
& \left\{y_{i}-g_{i}\left(x, D_{x}\right)+\text { lower order }\right\} K(x, y)=0 \quad(i=1, \cdots, n) .
\end{aligned}
$$

We can eliminate $D_{y_{i}}$ 's and $y_{i}$ 's from the lower order terms of (4.17) and get the isomorphism $(4 \cdot 16) .^{*)}$ Therefore principal symbols are invariant under a quantized contact transformation. But we cannot specify a unique quantized contact transformation for a given contact transformation.

If $M$ and $N$ are real, and $F: T^{*} M \rightarrow T^{*} N$ is a real contact transformation, the integral transformation defined by the kernel function $K(x$, y) $d y$ is an isomorphism from $\mathscr{C}_{N}$ to $\mathscr{C}_{M}$.

$$
\begin{aligned}
& \mathscr{C}_{W} \rightarrow \underset{\Psi}{\mathscr{C}_{M}} \\
& u(y) \mapsto \int K(x, y) u(y) d y .
\end{aligned}
$$

Exercise 4.18. Apply the formulas given in $\S 3$ to study $(4 \cdot 16)$.

Exercise 4.19. Show that these two isomorphisms of $\mathscr{E}$ and $\mathscr{C}$ are compatible.

* We call this isomorphism a quantized contact transformation. 
Example 4.20. Quantization of (4-12).

We take as a kernel function

$$
F_{G_{I_{0}-\{1,2\}}}(p-\widetilde{p}) d \widetilde{p}
$$

where $F_{G_{I_{0}-\{1,2\}}}(p)$ is the Feynman integral of the subgraph obtained from $(4 \cdot 8)$ by deleting two lines $k_{1}$ and $k_{2}$.

Then it transforms $\left.F_{G_{I_{0}-\{1,2\}}}\right|_{A_{I_{I_{0}-\{3,4\}}} \cup A_{p t, \tau}}$ into $\left.F_{G}\right|_{A_{G} \cup A_{G /\{1,2\}, \tau}}$.

Structure Theorem 4.21. Let $\mathcal{M}$ be a system of microdifferential equations with one generator. Assume that

(4.22) the characteristic variety $V$ is non-singular of codimensions $d(1 \leqq d \leqq n-1)$ and $\left.\sum_{i=0}^{n} \xi_{i} d x_{i}\right|_{V} \neq 0$, and that

(4.23) the symbol ideal $J$ is reduced.

Then $\mathcal{M}$ is transformed into

$$
D_{1} u=0, \cdots, D_{d} u=0 .
$$

To prove this theorem we first straighten the characteristic variety by a suitable contact transformation. Then by a corresponding quantized contact transformation the system is transformed into

$$
\left(D_{1}+Q_{1}(x, D)\right) v=0, \cdots,\left(D_{d}+Q_{d}(x, D)\right) v=0 .
$$

Then using the following two theorems $(4 \cdot 25)$ is reduced to $(4 \cdot 24)$.

Theorem 4.26. Let $P(x, D)$ and $Q(x, D)$ be microdifferential operators with the same principal symbol $P_{m}(x, \xi)$. Consider a point $\left(x_{0}, \xi_{0}\right)$ where $P_{m}(x, \xi)$ vanishes and assume that

$$
d_{(x, \xi)} P_{m}(x, \xi) \not X \sum_{i=1}^{n} \xi_{i} d x_{i} .
$$

Then there exists an elliptic microdifferential operator $R(x, D)$ such that

$$
R P R^{-1}=Q
$$

Theorem 4. 29. Let $P(x, D)$ be a microdifferential operator de- 
fined at $(0, \cdots, 0, \infty(1,0, \cdots, 0)) \in P^{*} X$. Assume that $\sigma(P)(0, \cdots, 0,1$, $\left.0, \cdots, \xi_{n}\right) / \xi_{n}{ }^{p}$ is holomorphic and never vanishes at $\xi_{n}=0$. Then any microdifferential operator $Q(x, D)$ can be divided by $P(x, D)$ with residue $R(x, D)=\sum_{j=1}^{p-1} R^{(j)}\left(x, D^{\prime}\right) D_{n}^{j}$ where $D^{\prime}=\left(D_{1}, \cdots, D_{n-1}\right)$.

$$
Q=S P+R \text {. }
$$

We shall explain structure theorems for holonomic systems and holonomic hyperfunctions.

(i) Let $\mathscr{M}$ be a holonomic system with support $\Lambda$. We assume that $\Lambda$ is non-singular and $\mathscr{M}$ is simple.

By a suitable contact transformation, $\Lambda$ is transformed into

$$
x_{1}=\xi_{2}=\cdots=\xi_{n} \text {. }
$$

But $(4 \cdot 27)$ fails to hold and the structure admits a parameter $\lambda$ and takes the form

$$
\varepsilon_{X} / \varepsilon_{X}\left(x_{1} D_{1}-\lambda\right)+\varepsilon_{X} D_{2}+\cdots+\varepsilon_{X} D_{n}^{*)} .
$$

(ii) Let $u(x)$ be a hyperfunction such that $\operatorname{sp} u$ satisfies a holonomic system $\mathscr{M}=\mathcal{E} u$ with support $\Lambda$, a conormal bundle of a hypersurface $\{f(x)=0\}$ where $\operatorname{grad} f(x) \neq 0$. We assume that $\mathscr{M}$ is simple and $\operatorname{ord}_{\Lambda}(u)=-\left(\lambda+\frac{1}{2}\right)$. We take $x_{1}=f(x)$, then by a suitable elliptic microdifferential operator $Q(x, D)$ of order 0 defined at $\left(0, d x_{1}\right) \in P^{*} X$ we can represent $\mathcal{M}=\mathcal{E v}_{v}$ as follows.

$$
\left(x_{1} D_{1}-\lambda\right) v=0, \quad D_{2} v=0, \cdots, D_{n} v=0,
$$

where $v=Q(x, D) u$.

Then using $(2 \cdot 8)$ and (1.56) we can determine the form of $u(x)$.

$$
\begin{aligned}
& \text { I. }(\lambda \notin Z) \quad F_{1}(x)(f(x)+i 0)^{\lambda}+F_{2}(x)(f(x)-i 0)^{\lambda}+F_{3}(x) \\
& \text { II. }(\lambda=0,1,2, \cdots) \quad F_{1}(x) f(x)^{\lambda} \log (f(x)+i 0) \\
& +F_{2}(x) f(x)^{\lambda} \log (f(x)-i 0)+F_{3}(x) \\
& \text { III. }(\lambda=-1,-2, \cdots) \quad F_{1}(x)(f(x)+i 0)^{\lambda}+F_{2}(x)(f(x)-i 0)^{2} \\
& +F_{3}(x) \log (f(x)+i 0)+F_{4}(x) \log (f(x)-i 0)
\end{aligned}
$$

* Different $\lambda$ 's give isomorphic systems if and only if the difference is an integer. If we use micro-differential operators of fractional order any $\lambda$ gives the same system. 
where $F_{1}(x)$ and $F_{2}(x)$ are real analytic and either $\equiv 0$ or $\neq 0, F_{3}(x)$ and $F_{4}(x)$ are real analytic.

\section{Example $4 \cdot 25$.}

We have transformed

$$
\left.F_{G}\right|_{\Lambda_{G} \cup \Lambda_{G /\{1,2\}, \tau}} \text { to }\left.F_{G_{I_{0}-\{3,4\}}}\right|_{A_{G_{I_{0}-\{3,4\}}}} \cup_{A_{\mathrm{pt}, \tau}}
$$

in $(4 \cdot 20)$. Now we can determine the structure of $F_{G_{I_{0}-\{3,4\}}}$.

In general $F_{G}(p)$ satisfies a simple holonomic system of order

$$
\operatorname{ord}_{\Lambda_{G}} F_{G}=\frac{1}{2}\{N+\nu(n-N)\}
$$

where $N=\{$ internal lines $\}$ and $n=\neq$ vertices $\}$. We can write

$$
F_{G_{I_{0}-\{3,4\}}}\left(\widetilde{p}_{1}, \widetilde{p}_{2}, \widetilde{p}_{3}\right)=f\left(\widetilde{p}_{1}\right) \delta\left(\widetilde{p}_{1}+\widetilde{p}_{2}\right) \delta\left(\widetilde{p}_{3}\right) .
$$

Then $f\left(\widetilde{p}_{1}\right)$ is a holonomic hyperfunction with singular specturm in

$$
\left\{\left(\widetilde{p}_{1}, \sqrt{-1} \infty \widetilde{p}_{11} \cdot d \widetilde{p}_{11}\right) \mid \widetilde{p}_{1}^{2}=\left(m_{1}+m_{2}\right)^{2}\right\}
$$

and of order $1-\frac{\nu}{2}$ or $\lambda=\frac{\nu-3}{2}$.

Thus we have one of the canonical forms of $(4 \cdot 24)$, where $F_{2}(x)$ $\equiv 0 . *$

Similarly the structure of a Feynman amplitude at a generic point of any intersection $\Lambda_{G / I_{1}, \tau} \cup \Lambda_{G / I_{1} \cup I_{2}, \tau}$ of codimension 1 is one of the forms I III with $F_{2}(x) \equiv 0$. $^{*}$

(iii) Let $\mathscr{M}$ be a holonomic system with support $\Lambda=\Lambda_{1} \cup \Lambda_{2}$. We assume that $\Lambda_{1}, \Lambda_{2}$ and $\Lambda_{1} \cap \Lambda_{2}$ are non-singular and $\Lambda_{1} \cap \Lambda_{2}$ has dimensions $n-1$. Moreover we assume that $T_{p}\left(\Lambda_{1} \cap \Lambda_{2}\right)=\left(T_{p} \Lambda_{1}\right) \cap\left(T_{p} \Lambda_{2}\right)$ for any $p \in \Lambda_{1} \cap \Lambda_{2}$, and the symbol ideal $J$ is reduced. Then $\mathscr{M}$ is transformed to Example (2.53). In (2.53) $\beta=\operatorname{ord}_{\Lambda_{2}}(u)-\operatorname{ord}_{\Lambda_{1}}(u)-\frac{1}{2}$ has an essential meaning. According to the value of $\beta, \mathscr{M}$ is classified into three classes. This we have already explained in Summarize $(1 \cdot 30)$ by examining the solution space, where we use $\lambda$ in place of $\beta$.

Now as a final remark we explain the difference of their algebraic properties. (The proof is straightforward.)

$*$ In case III, $F_{4}(x) \equiv 0$. 


\section{Theorem 4.29.}

I. If $\beta \notin Z, \mathscr{M}$ is an irreducible $\mathcal{E}_{X}$-Module.

II. If $\beta=0,1,2, \cdots$ there is an exact sequence of $\varepsilon_{X}$-Modules

$$
0 \rightarrow \mathscr{M}_{1} \rightarrow \mathscr{M} \rightarrow \mathscr{N}_{2} \rightarrow 0
$$

where supp $\mathcal{M}_{i} \subset \Lambda_{i}(i=1,2)$.

III. If $\beta=-1,-2, \cdots$ there is an exact sequence of $\mathcal{E}_{X}$-Modules

$$
0 \rightarrow \mathscr{M}_{2} \rightarrow \mathscr{M} \rightarrow \mathscr{M}_{1} \rightarrow 0
$$

where supp $\mathscr{M}_{i} \subset \Lambda_{i}(i=1,2)$. 